\title{
Does the Endothelium of Competitive Athletes Benefit from Consumption of n-3 Polyunsaturated Fatty Acid-Enriched Hen Eggs?
}

\author{
Luka Kolar ${ }^{1,2 *}$, Marko Stupin ${ }^{2,3,4 *}$, Ana Stupin ${ }^{2,4}$, Petar Šušnjara ${ }^{2,4}$, Zrinka Mihaljević $^{2,4}$, Anita Matić, ${ }^{2,4}$, \\ Ivana Jukić ${ }^{2,4}$, Nikolina Kolobarić, ${ }^{2,4}$, and Ines Drenjančević ${ }^{2,4}$ \\ ${ }^{1}$ Department of Internal Medicine, National Memorial Hospital Vukovar, Vukovar 32000, Croatia \\ ${ }^{2}$ Scientific Center of Excellence for Personalized Health Care and ${ }^{4}$ Department of Physiology and Immunology, \\ Faculty of Medicine, Josip Juraj Strossmayer University of Osijek, Osijek 31000, Croatia \\ ${ }^{3}$ Department for Cardiovascular Disease, Osijek University Hospital, Osijek 31000, Croatia
}

\begin{abstract}
The present study aimed to determine the effect of $n-3$ polyunsaturated fatty acid (PUFA)-enriched hen eggs on microvascular vasodilation, microvascular responsiveness to a stress challenge and markers of oxidative stress in competitive athletes. Competitive athletes $(n=23)$ were divided to a control group $(n=9)$, who consumed three regular hens' eggs daily (249 mg n-3 PUFAs/d), and n-3 PUFAs group ( $n=14)$, who consumed three $n-3$ PUFA-enriched hen eggs daily (1,053 g n-3 PUFAs/d) for 3 weeks. Endothelium-dependent responses [post-occlusive reactive hyperemia (PORH) and acetylcholine-induced dilation (AChID)] and endothelium-independent responses [sodium nitroprusside-induced dilation (SNPID)] of skin microvascular blood flow were assessed by laser Doppler flowmetry in pre- and post-acute exhausting exercise (AEE) sessions. Blood pressure, serum lipid, free fatty acids profiles, and biomarkers of oxidative stress were measured before and after each dietary protocol. Consumption of serum n-3 PUFAs significantly decreased the n-6/n-3 ratio and enhanced PORH and AChID, but did not affect SNPID at rest. Furthermore, serum glutathione peroxidase and superoxide dismutase activities were significantly decreased in the n-3 PUFAs group but remained unchanged in the control group. In both groups, PORH, AChID, and SNP were significantly reduced post-AEE compared with pre-AEE, both before and after consumption of each diet. Only AChID responsiveness to AEE ( $\triangle \mathrm{AChID}$ ) significantly increased following consumption of n-3 PUFAs. Overall, n-3 PUFAs supplementation as n-3 PUFA-enriched hen eggs enhanced microvascular endothelial function at rest and may contribute to adaptation to AEE in competitive athletes.
\end{abstract}

Keywords: athletes, endothelium, microcirculation, n-3 polyunsaturated fatty acids, oxidative stress

\section{INTRODUCTION}

Proper nutrition is necessary for competitive athletes to adjust to high physical and metabolic activity due to increased energy and nutritional needs (Thielecke and Blannin, 2020). The interest in n-3 polyunsaturated fatty acids (PUFAs) in sport nutrition is rising, due to their potential to preserve cardiovascular (CV) health and prevent progression of CV diseases (Thies et al., 2003; Zehr and Walker, 2018; Stupin et al., 2019). n-3 PUFAs may exert these effect through decreasing blood pressure (BP) (Appel et al., 1993), enhancing anti-inflammatory poten- tial (Calder, 2006), reducing formation of atherosclerotic lesions (Renier et al., 1993), increasing antioxidant capacity (Thorlaksdottir et al., 2006), and altering the balance between endothelium-derived vasodilators and vasoconstrictors that result in improved endothelium-dependent vasodilation (Drenjančević et al., 2017). Interestingly, analyses of dietary habits in athletes found that a substantial proportion do not reach dietary goals for macro- and micronutrients, including of n-3 PUFAs such as eicosapentaenoic acid (EPA) and docosahexaenoic acid (DHA) (von Schacky et al., 2014; Wilson and Madrigal, 2016; Ritz et al., 2020). Since hen eggs are a cheap source

Received 27 July 2021; Revised 25 October 2021; Accepted 3 November 2021; Published online 31 December 2021

Correspondence to Ines Drenjančević, Tel: +38-531512800, E-mail: ines.drenjancevic@mefos.hr,

Ana Stupin, Tel: +38-531512869, E-mail: anacavka@mefos.hr

*These authors contributed equally to this work.

Author information: Luka Kolar (Researcher), Marko Stupin (Researcher), Ana Stupin (Professor), Petar Šušnjara (Researcher), Zrinka Mihaljević (Professor), Anita Matić (Professor), Ivana Jukić (Professor), Nikolina Kolobarić (Researcher), Ines Drenjančević (Professor) 
of essential nutrients and energy, consumption of eggs is very accepted by athletes. Therefore, eggs enriched with n-3 PUFAs could present a nutritionally rich and natural way of supplementing n-3 PUFAs in athletes, especially since previous research has not shown a detrimental effect of daily egg consumption on lipid profiles, liver function, or general health in healthy sedentary populations (Stupin et al., 2018a).

It is generally recommended to consume n-3 PUFAs via seafood (rich in EPA/DHA), since humans EPA can be synthesized from alpha-linolenic acid (ALA) but EPA is only converted to DHA at a low rate (Arterburn et al., 2006). Since pharmacological supplements containing n-3 PUFAs may be banned by sport associations and federations (Oliver, 2019), development of functional foods rich in n-3 PUFAs is both of scientific and commercial interest for athletes (Drenjančević et al., 2017). Most commonly produced n-3 PUFA-enriched functional foods include poultry products (e.g., hen eggs and chicken meat), fortified pasta, soy milk, oatmeal, cereal, and margarine (Drenjančević et al., 2017). Consumption of n-3 PUFA is beneficial for vascular reactivity and biochemical blood markers in young individuals. For example, consumption of five n-3 PUFAs enriched hen eggs (241 mg n-3 PUFA/egg) per week for three weeks significantly decreased serum triglycerides levels $(16 \% \sim 18 \%$ decrease) in 25 healthy individuals (Bovet et al., 2007). Similar, we previously showed that consumption of three n-3 PUFA-enriched hen eggs per day (777 mg or 1,053 mg n-3 PUFA/d) for three weeks enhanced skin microvascular post-occlusive hyperemic responses and decreased arterial BP, blood triglycerides, and high-sensitivity C-reactive proteins (hsCRPs) in young healthy subjects (Stupin et al., 2018a; Stupin et al., 2020), and decreased serum pro-inflammatory [e.g., interferon- $\gamma$ and increased serum anti-inflammatory cytokines (interluekin-10) in young healthy sedentary individuals]. Therefore, n-3 PUFAs may have anti-inflammatory properties that are beneficial for vascular and endothelial function (Stupin et al., 2020).

Regular exercise preserves $\mathrm{CV}$ health and improves endothelium-dependent macro- and microvascular vasodilation in healthy individuals, helping to alleviate endothelial dysfunction and inflammation in the vascular wall of CV patients. These effects result from preventive effects on traditional CV risk factors (e.g., BP, blood lipids) and improved vascular reactivity (Joyner and Green, 2009; Dawson et al., 2013). However, acute strenuous exercise presents great mechanical and CV stress, which may have a transient but significant impact on the body (Jurva et al., 2006; Stupin et al., 2018b). Although many sports (e.g., short-distance running, weight lifting, etc.) involve short-term vigorous efforts resulting in acute exertion, the effects of acute exertions on vascular function and the long-term adjustments of the vascular system to regular exercise have not been elucidated. It has been suggested that acutely increased oxidative stress level following intense bouts of acute exercise results in the long-term up-regulation of the body's antioxidant defense mechanisms observed in the athletes who exercise regularly (Powers and Jackson, 2008). Previously, we demonstrated that a single acute exercise session impairs microvascular reactivity and endothelial function in professional rowers. At baseline the athletes had enhanced microvascular endothelial function compared with sedentary controls. However, the athletes had higher increases in arterial pressure and more unfavorable oxidative milieu following acute exercise than sedentary individuals (Stupin et al., 2018b). These findings support the 'hormesis' hypothesis, which suggests that long-term improvement of physiological function to a repeated stimulus (e.g., exercise training) can be induced even when this stimulus temporarily impairs the physiological system involved (Dawson et al., 2013).

The evidence suggests that both n-3 PUFAs and regular exercise have potential to improve macro- and microvascular endothelial function via similar mechanisms in healthy individuals (and athletes), and individuals with increased CV risk or patients with CV disease (Morgan et al., 2006; Joyner and Green, 2009; Egert et al., 2014). However, there is a paucity of data on the interactive effect of these two factors on endothelial function. Thus, the present study was designed to: a) determine the effect of consumption of n-3 PUFA-enriched hen eggs on endothelium-dependent and -independent vasodilation of forearm skin microcirculation; b) test if n-3 PUFAs functional food consumption modifies microvascular adaptation, i.e., reactivity responses to stress challenges in acute exhausting exercise (AEE) sessions; and c) examine the effect of n-3 PUFA consumption on measurable markers of oxidative stress and antioxidant defense in competitive athletes.

\section{MATERIALS AND METHODS}

\section{Study population}

Twenty-three young healthy competitive athletes (3 females and 20 males) participated in this study. Athletes were recruited from local rowing, football, and athletics sports clubs. All athletes had been training 5 to 12 times a week for at least 5 years. Further eligibility criteria included age of 18 to 30 years, and normal body mass index (BMI), arterial BP, and serum lipid ranges. Exclusion criteria included a history of smoking, hypertension, coronary artery disease, diabetes, hyperlipidemia, renal impairment, cerebrovascular and peripheral artery disease, and taking any drugs or substances that could affect the 
endothelium (e.g., oral contraceptives in female). Also, participants must not have been taking n-3 PUFA-enriched functional food or n-3 PUFA supplementation in the form of capsules prior to enrollment in the present study. Written informed consent was obtained from each subject. The study protocol and procedures conformed with the standards set by the latest revision of the Declaration of Helsinki and were approved by the Ethical Committee of the Faculty of Medicine, University of Osijek, Osijek, Croatia (Class: 602-04/19-09/7; No: 38360-0119-13).

\section{Production of n-3 PUFA-enriched hen eggs and assessment of fatty acid profiles of chicken feed mixtures and edible part of eggs}

n-3 PUFA-enriched hen eggs were produced according to the protocol of research group from Faculty of Agrobiotechnical Sciences, University of Osijek (Stupin et al., 2018a; Stupin et al., 2020; Mihalj et al., 2020). In brief, soybean oil (5\%) fed to laying hens in feed was replaced with a mixture of fish (1.5\%) and linseed (3.5\%) oil. Preparation and analysis of samples (feeding mixtures for laying hens and edible parts of eggs) to determine fatty acid content was performed in accordance with our wellestablished protocols, which are described in detail in previous publications (Stupin et al., 2018a; Stupin et al., 2020; Mihalj et al., 2020). Portions of saturated fatty acid, monounsaturated fatty acid, n-6 PUFAs, and n-3 PUFAs were determined in $\mathrm{g} / 100 \mathrm{~g}$ of total fatty acids in feed, and in $\mathrm{mg} / 100 \mathrm{~g}$ in eggs (edible part), as previously described (Stupin et al., 2020). The n-6/n-3 PUFA ratio in feed for hens that produced regular eggs was 10.30, and for those that produced n-3 PUFAs eggs was 0.69. Each n-3 PUFAs egg (average weight $60 \mathrm{~g}$ ) contained an average of $351 \mathrm{mg}$ of n-3 PUFAs (ALA $230.5 \mathrm{mg} / \mathrm{egg}$, EPA $15.1 \mathrm{mg} / \mathrm{egg}$, and DHA $105.5 \mathrm{mg} / \mathrm{egg})$. Each control egg (average weight $60 \mathrm{~g}$ ) contained an average of $83 \mathrm{mg}$ of n-3 PUFAs (ALA $36 \mathrm{mg} / \mathrm{egg}$, EPA $0 \mathrm{mg} / \mathrm{egg}$, and DHA $47 \mathrm{mg} / \mathrm{egg})$. The $\mathrm{n}-6 / \mathrm{n}-3$ PUFAs ratio in n-3 PUFA hen eggs (ratio 2.02) was significantly more favorable than in regular hen eggs (ratio 10.26).

\section{Study design}

This was a randomized placebo-controlled interventional study. To reduce the risk of bias, assessors of anthropometric, $\mathrm{CV}$, biochemical and functional vascular outcomes were blinded. The study protocol lasted for three weeks (21 day), during which subjects were instructed to eat three hard boiled hen eggs per day (total of 63 eggs). Subjects were divided into the experimental n-3 PUFA group ( $\mathrm{n}=14,3$ females and 11 males) who consumed n-3 PUFA-enriched hen eggs (3 per day; approximately $1,053 \mathrm{mg}$ of n-3 PUFA per day), and the control group $(n=9,0$ female and 9 males) who consumed regular hen eggs produced on the same farm (3 per day; approximately 249 mg of n-3 PUFA per day). n-3 PUFA-enriched eggs and regular hen eggs were the same size (commercial size: medium), and neither the researcher or the subjects knew which group each subject was in. Furthermore, all subjects were instructed to consume only the eggs given to them for the purposes of the study and not to consume other foods rich in n-3 PUFAs, n-3 PUFAenriched functional food or any other form of n-3 PUFAs supplementation during the study. The study was performed in the Laboratory for Clinical and Sport Physiology, Department of Physiology and Immunology at the Faculty of Medicine, University of Osijek, Croatia. Two study visits and all measurements were conducted on the first day and the day immediately after the end of the protocol. All testing occurred in the morning after overnight fasting. Participants were instructed not to undertake any strenuous activity during the 24 -h preceding the visits. The study protocol is depicted in the Fig. 1.

Basic anthropometric, $\mathrm{CV}$, and biochemical measurements BMI was calculated by measuring subjects' heights (m) and weights $(\mathrm{kg})$, and waist and hip circumferences were measured to calculate waist-to-hip ratios (WHRs). BP and heart rate (HR) were measured at the beginning of each visit after a 15 min rest in a seated position using an

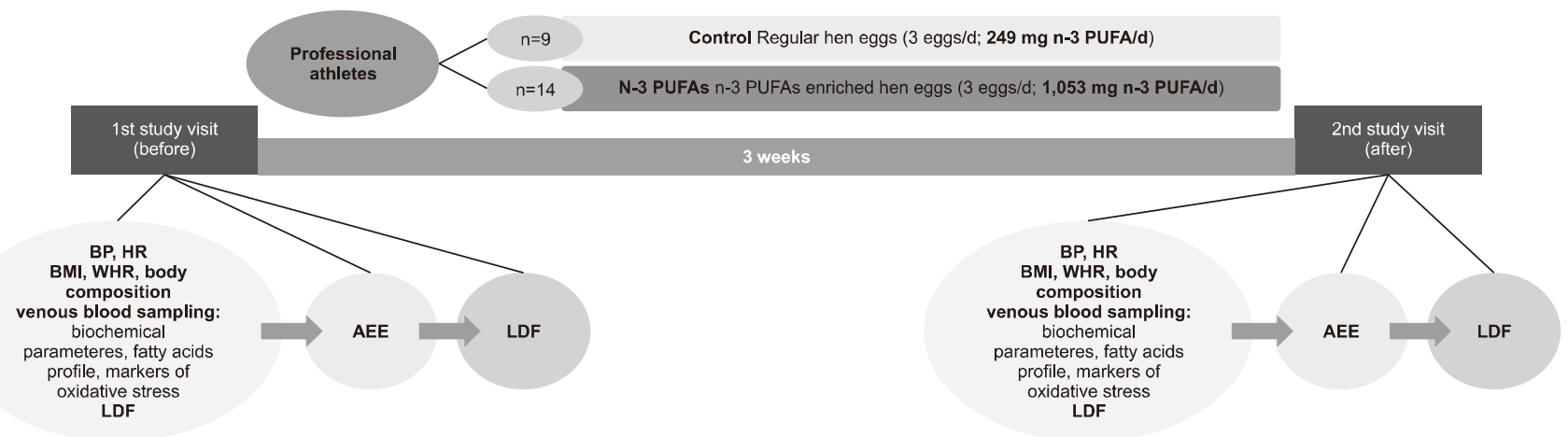

Fig. 1. Study timeline and protocol. PUFA, polyunsaturated fatty acid; BP, blood pressure; HR, heart rate; BMI, body mass index; WHR, waist-to-hip ratio; LDF, laser Doppler flowmetry; AEE, acute exhausting exercise session. 
automated oscillometric sphygmomanometer (OMRON M3, OMRON Healthcare Inc., Osaka, Japan). The final BP and $\mathrm{HR}$ values were determined as the mean of three repeated measurements. Venous blood samples were taken after a 15 min rest in a seated position at each study visit. Standard biochemical measurements involved blood sample analyses for full blood count, plasma electrolytes (sodium and potassium), urea, creatinine, fasting lipid profile [total cholesterol, high-density lipoprotein (HDL) cholesterol, low-density lipoprotein (LDL) cholesterol, and triglycerides], fasting blood glucose, hsCRP (a marker of inflammation), and von Willebrand factor (vWf; a marker of endothelial damage) were determined using standard laboratory methods, performed at the Department of Clinical Laboratory Diagnostics, University Hospital Osijek, Osijek, Croatia.

\section{Analysis of serum fatty acids profile}

Gas chromatography-tandem mass spectrometry (GCMS/MS) was used to analyze 37 fatty acids in serum, conducted in accordance with well-established protocols, which have been previously described in detail (Stupin et al., 2020). In brief, fatty acids were identified using the GC-MS/MS system by Thermo Fisher GC Trace 1300 coupled with a TSQ 9000 Triple Quadrupole (Thermo Fisher Scientific, Waltham, MA, USA), and the fatty acids profile was analyzed at the BIOCentre's Bioanalytical Laboratory, BIOCentre-Incubation Center for Biosciences, Zagreb, Croatia.

\section{Response of microvascular function to challenge- AEE session protocol}

To examine if the dietary protocol of this study (consumption of n-3 PUFA-enriched eggs vs. regular hen eggs) affects microvascular function to a given challenge (load), subjects underwent AEE in the form of rowing at each study visit. The AEE comprised of a progressively more intense rowing protocol that was modified to comprise $5 \times 4$-min submaximal grades and a single maximal grade (Stupin et al., 2018b). The rowing protocol was performed on the Dynamic Indoor Rower Concept 2 rowing ergometer (Concept2 Inc., Morrisville, VT, USA). All subjects were instructed on how to use the rowing ergometer by a former professional rower, and each practiced once before the AEE. Submaximal grades were determined by the given load and lasted for 4 min each. The exercise session started with an initial workload of 150 $\mathrm{W}$, with stage progression of $40 \mathrm{~W}$. The submaximal grades were separated by 1-min recovery periods, with a 5 -min rest before the maximal stage. Subjects were instructed to row with maximum power until complete exhaustion with no time limitation. Rating of perceived exertion was used for subjective quantification of individuals' perceptions of the physical demands of the activity.
In this study, a category-ratio scale (CR10), introduced by Borg (1982) was used. CR10 uses rating ranging from 0 (nothing) to 10 (very hard activity), and shows good reliability and validity in healthy, clinical, and athletic adult populations (Chen et al., 2002).

\section{Assessment of skin microcirculatory blood flow}

Laser Doppler flowmetry (LDF) (MoorVMS-LDF, Moor Instruments Ltd., Axminster, UK) was used to assess overall microvascular reactivity by induction of post-occlusive reactive hyperemia (PORH), and to evaluate endothelium-dependent and -independent vasodilation by iontophoresis (noninvasive transdermal application of charged substances) of acetylcholine (ACh) and sodium nitroprusside (SNP), respectively. The procedures for LDF measurements have been previously described in detail (Cavka et al., 2015; Barić et al., 2019). The PORH test included forearm microvascular blood flow measurements before, during, and after release of 1-min vascular occlusion. Microcirculatory blood flow was determined by software calculating the area under the curve (AUC) during baseline flow, occlusion, and reperfusion, and results were expressed as differences between the percentage of flow change during reperfusion and occlusion in relation with baseline (R-O\% increase). After baseline microvascular blood flow recording, either the positively charged ACh (1\%) or negatively charged SNP (1\%) vasodilator was subjected to iontophoresis with an anodal current, applied by following an established protocol, adapted to obtain a stable plateau of the maximal LDF response (Stupin et al., 2018b; Barić et al., 2019). Microcirculatory blood flow was determined using software to calculate the AUC during baseline flow and during ACh or SNP administration. Results were expressed as increases in blood flow following ACh or SNP administration in relation to baseline flow (ACh or SNP blood flow increase).

\section{Assessment of microvascular responsiveness range to AEE}

Microvascular responsiveness range to AEE was calculated according to a previously established protocol (Stupin et al., 2018b). Microvascular responsiveness ranges are measures of difference in microvascular reactivity responses to vascular occlusion (PORH), ACh (acetylcholine-induced dilation, AChID), and SNP (sodium nitroprusside-induced dilation, SNPID) administration, assessed pre- and post-AEE sessions, and is expressed as $\triangle \mathrm{PORH}, \triangle \mathrm{AChID}$, and $\Delta$ SNPID. Delta $(\Delta)$ values were obtained at each study visit (before and after completing each diet protocol) by subtracting the result of each measurement (PORH, AChID, or SNP) after AEE from the measurement before AEE $\left(\triangle \mathrm{PORH}=\mathrm{PORH}_{\text {post-AEE }}-\right.$ $\mathrm{PORH}_{\text {pre-AEE}} ; \quad \triangle \mathrm{AChID}=\mathrm{AChID}_{\text {post-AEE }}-\mathrm{AChID}_{\text {pre-AEE}} ;$ $\left.\Delta \mathrm{SNPID}=\mathrm{SNPID}_{\text {post-AEE }}-\mathrm{SNPID}_{\text {pre-AEE }}\right)$. 
Measurement of oxidative stress, antioxidant capacity, and antioxidant enzymes activity

The thiobarbituric acid reactive substances (TBARS) method measures products of lipid peroxidation and is a measure of oxidative stress. Absorbance of samples are measured by NanoPhotometer P300 (Implen GmbH, München, Germany) at 572 and $532 \mathrm{~nm}$, with malondialdehyde (MDA) used as a standard ( $\mu \mathrm{M}$ MDA). The ferric-reducing ability of plasma (FRAP) method was used to measure antioxidant capacity of blood samples. $\mathrm{Fe}^{3+}$ 2,4,6-tris(2-pyridyl)-s-triazine (TPTZ) is reduced to $\mathrm{Fe}^{2+}$ TPTZ in the presence of antioxidants to produce blue discoloration. The absorbance of the samples was measured using a NanoPhotometer P300 (Implen) at $593 \mathrm{~nm}$ (mM/L Trolox). TBARS and FRAP measurements were conducted according to previously described protocols (Barić et al., 2020). Enzyme activity of catalase (CAT), glutathione peroxidase (GPx), and superoxide dismutase (SOD) in serum was performed using a Lambda 25UVVis spectrophotometer equipped with the UV WinLab 6.0 software package (PerkinElmer For the Better, Waltham, MA, USA), according to the protocol established in the Biochemistry Laboratory at the Department of Biology,
University of Osijek (Cosic et al., 2016; Mihaljević et al., 2018). The activities of CAT, GPx, and SOD were expressed as units of the enzymes per milligram of protein ( $\mathrm{U} / \mathrm{mg}$ protein). Concentrations of proteins in serum $(\mathrm{mg} / \mathrm{mL})$ were determined using Bradford reagent (Bradford Reagent B6916, Sigma Aldrich Co., St. Louis, MO, USA) following the manufacturer's protocol, measured at $595 \mathrm{~nm}$ using bovine serum albumin as a standard.

\section{Statistical analysis}

All results are reported as the arithmetic mean \pm standard deviation. The sample size required to show a potentially significant effect was calculated based on preliminary data collected from 8 subjects. To detect differences in primary outcomes reported in this study (e.g., LDF measurement) with a level of significance of 0.05 and a statistical power of $80 \%$ for paired $t$-tests, a minimum sample size of 10 subjects per group was required. The normality of data distribution was assessed by Kolmogorov-Smirnov normality tests. Differences between measurements before and after each diet protocol were assessed using paired $t$-tests, or Wilcoxon rank-sum tests when variables were not normally distributed. To assess

Table 1. The effect of regular (control group) and n-3 PUFA-enriched ( $n-3$ PUFAs group) hen egg consumption on anthropometric, hemodynamic, and biochemical parameters

\begin{tabular}{|c|c|c|c|c|}
\hline \multirow{2}{*}{ Parameter } & \multicolumn{2}{|c|}{ Control } & \multicolumn{2}{|c|}{ n-3 PUFA } \\
\hline & Before & After & Before & After \\
\hline$N(F / M)$ & \multicolumn{2}{|c|}{$9(0 / 9)$} & \multicolumn{2}{|c|}{$14(3 / 11)$} \\
\hline Age (yr) & \multicolumn{2}{|c|}{$22 \pm 2$} & \multicolumn{2}{|c|}{$22 \pm 3$} \\
\hline BMI $\left(\mathrm{kg} / \mathrm{m}^{2}\right)$ & $24.7 \pm 1.6$ & $24.7 \pm 1.7$ & $23.5 \pm 2.1$ & $23.4 \pm 2.1$ \\
\hline WHR & $0.86 \pm 0.05$ & $0.85 \pm 0.07$ & $0.81 \pm 0.05$ & $0.81 \pm 0.05$ \\
\hline $\mathrm{SBP}(\mathrm{mmHg})$ & $120 \pm 8$ & $114 \pm 13$ & $123 \pm 13$ & $121 \pm 12$ \\
\hline $\mathrm{DBP}(\mathrm{mmHg})$ & $72 \pm 8$ & $66 \pm 12$ & $73 \pm 7$ & $70 \pm 7$ \\
\hline MAP (mmHg) & $88 \pm 7$ & $82 \pm 11$ & $89 \pm 8$ & $87 \pm 8$ \\
\hline HR (beat/min) & $80 \pm 12$ & $74 \pm 18$ & $66 \pm 11$ & $70 \pm 14$ \\
\hline Erythrocytes $\left(\times 10^{\mathrm{e} 12} / \mathrm{L}\right)$ & $4.9 \pm 0.3$ & $4.9 \pm 0.3$ & $4.9 \pm 0.4$ & $4.9 \pm 0.3$ \\
\hline Hemoglobin $(g / L)$ & $146 \pm 6$ & $147 \pm 6$ & $147 \pm 9$ & $145 \pm 7$ \\
\hline Hematocrit (\%) & $41.9 \pm 1.8$ & $42.3 \pm 1.6$ & $42.5 \pm 2.0$ & $40.8 \pm 2.8$ \\
\hline Leukocytes $\left(\times 10^{\mathrm{eq}} / \mathrm{L}\right)$ & $6.6 \pm 1.1$ & $7.0 \pm 1.8$ & $6.4 \pm 2.2$ & $6.3 \pm 2.7$ \\
\hline Thrombocytes $\left(\times 10^{\mathrm{eq}} / \mathrm{L}\right)$ & $204 \pm 28$ & $217 \pm 42$ & $209 \pm 48$ & $207 \pm 44$ \\
\hline Urea $(\mathrm{mmol} / \mathrm{L})$ & $6.9 \pm 1.4$ & $7.1 \pm 1.2$ & $6.5 \pm 1.3$ & $6.8 \pm 1.4$ \\
\hline Creatinine $(\mu \mathrm{mol} / \mathrm{L})$ & $90 \pm 7$ & $89 \pm 8$ & $88 \pm 16$ & $85 \pm 15$ \\
\hline Sodium (mmol/L) & $137.9 \pm 2.2$ & $139.8 \pm 2.8$ & $137.5 \pm 2.1$ & $137.9 \pm 2.2$ \\
\hline Potassium (mmol/L) & $4.3 \pm 0.4$ & $4.2 \pm 0.3$ & $3.9 \pm 0.6$ & $3.9 \pm 0.5$ \\
\hline Glucose (mmol/L) & $4.9 \pm 0.8$ & $5.1 \pm 1.4$ & $4.8 \pm 0.5$ & $4.8 \pm 0.5$ \\
\hline hsCRP (mg/L) & $0.67 \pm 1.09$ & $0.90 \pm 1.25$ & $0.72 \pm 1.11$ & $1.15 \pm 1.39$ \\
\hline von Willebrand factor & $1.26 \pm 0.14$ & $1.45 \pm 0.30$ & $1.37 \pm 0.59$ & $1.33 \pm 0.46$ \\
\hline Total cholesterol (mmol/L) & $4.4 \pm 0.4$ & $4.4 \pm 0.7$ & $4.4 \pm 0.4$ & $4.7 \pm 0.5$ \\
\hline Triglycerides (mmol/L) & $1.0 \pm 0.5$ & $1.4 \pm 0.7$ & $1.0 \pm 0.5$ & $1.1 \pm 0.5$ \\
\hline HDL cholesterol (mmol/L) & $1.4 \pm 0.2$ & $1.4 \pm 0.3$ & $1.4 \pm 0.2$ & $1.5 \pm 0.2$ \\
\hline LDL cholesterol (mmol/L) & $2.5 \pm 0.2$ & $2.5 \pm 0.4$ & $2.5 \pm 0.2$ & $2.7 \pm 0.4$ \\
\hline
\end{tabular}

Values are presented as mean \pm SD.

PUFA, polyunsaturated fatty acid; N, number of participants; $F$, female; $M$, male; BMI, body mass index; WHR, waist-to-hip ratio; $\mathrm{SBP}$, systolic blood pressure; DBP, diastolic blood pressure; MAP, mean arterial pressure; HR, heart rate; hsCRP, high-sensitivity $C$ reactive protein; $H D L$, high-density lipoprotein; $L D L$, low-density lipoprotein. 
differences between control and n-3 PUFA groups, Student's $t$-tests were used for parametric distributions and Mann-Whitney tests were used for nonparametric distributions. Correlations between primary outcomes (microvascular reactivity; e.g., PORH, AChID, and SNPID) and corresponding parameters (BP, HR, BMI, WHR, hsCRP, serum lipids, TBARS, FRAP, and antioxidant enzymes activity) in both study groups were determined by Pearson's or Spearman's correlation tests, as appropriate. $P<0.05$ was considered statistically significant. SigmaPlot version 11.2 (Systat Software Inc., Chicago, IL, USA) was used for statistical analysis.

\section{RESULTS}

Subjects' baseline anthropometric, hemodynamic, and biochemical characteristics are presented in Table 1. All subjects were lean and normotensive, had normal full blood counts, renal function, serum electrolytes, fasting blood glucose, hsCRP, and fasting lipid levels. At baseline, there was no significant difference in all measured parameters (e.g., age, BMI, BP, HR, and biochemical parameters) between athletes in the control and n-3 PUFA groups. All participants completed the 3-weeks dietary protocol: the n-3 PUFAs group consumed n-3 PUFA-enriched hen eggs ( 3 eggs with a total of 1,053 n-PUFAs/d) and the control group consumed regular hen eggs (3 eggs with a total of 249 n-PUFAs/d).

\section{Anthropometric, CV, and biochemical parameters}

The effects of consuming regular or n-3 PUFA-enriched hen eggs on anthropometric, hemodynamic and biochemical parameters are presented in Table 1 . There were no significant differences in anthropometric (BMI, WHR), hemodynamic (systolic BP, diastolic BP, mean arterial pressure, and HR), or biochemical (full blood count, plasma electrolytes, urea, creatinine, fasting blood glucose, hsCRP, and vWf) parameters, or in serum lipid profiles (total cholesterol, triglycerides, LDL cholesterol, and HDL cholesterol) following consumption of $n-3$ PUFA or regular hen eggs compared with baseline measurements. Furthermore, these measurements did not differ between groups.

Table 2. The effect of regular (control group) and n-3 PUFA-enriched ( $n-3$ PUFAs group) hen egg consumption on serum fatty acids profiles

\begin{tabular}{|c|c|c|c|c|}
\hline \multirow{2}{*}{ Parameter } & \multicolumn{2}{|c|}{ Control } & \multicolumn{2}{|c|}{ n-3 PUFAs } \\
\hline & Before & After & Before & After \\
\hline \multicolumn{5}{|l|}{ SFA ( $\mu \mathrm{mol} / L)$} \\
\hline C14:0 Myristic acid & $30.5 \pm 0.8$ & $36.9 \pm 19.1$ & $28.3 \pm 4.1$ & $24.0 \pm 7.6$ \\
\hline C16:0 Palmitic acid & $376 \pm 79$ & $591 \pm 287$ & $341 \pm 97$ & $337 \pm 62$ \\
\hline C18:0 Stearic acid & $115 \pm 18$ & $172 \pm 69$ & $101 \pm 25$ & $105 \pm 20$ \\
\hline \multicolumn{5}{|l|}{ PUFA ( $\mu \mathrm{mol} / \mathrm{L})$} \\
\hline \multicolumn{5}{|l|}{$n-7$} \\
\hline C16:1(cis-9) Palmitoleic acid & $26.9 \pm 5.1$ & $43.9 \pm 23.3$ & $28.9 \pm 7.1$ & $27.5 \pm 1.5$ \\
\hline \multicolumn{5}{|l|}{$\mathrm{n}-9$} \\
\hline 18:1(cis-9) Oleic acid & $298 \pm 142$ & $496 \pm 257$ & $214 \pm 71$ & $212 \pm 40$ \\
\hline \multicolumn{5}{|l|}{$n-6$} \\
\hline C18:2(cis-9,12) Linoleic acid & $597 \pm 132$ & $903 \pm 337$ & $460 \pm 144$ & $468 \pm 129$ \\
\hline C18:3(cis-6,9,12) Gamma-Linolenic acid & $12.7 \pm 4.2$ & $13.4 \pm 2.0$ & $12.1 \pm 0.3$ & $11.5 \pm 0.1$ \\
\hline C20:3(cis-8,11,14) Dihomo-gamma-linolenic acid & $23.6 \pm 4.2$ & $28.9 \pm 9.3$ & $23.9 \pm 6.0$ & $23.3 \pm 8.4$ \\
\hline C20:4(cis-5,8,11,14) Arachidonic acid & $161 \pm 51$ & $224 \pm 58^{\dagger}$ & $128 \pm 25$ & $126 \pm 21$ \\
\hline \multicolumn{5}{|l|}{$\mathrm{n}-3$} \\
\hline C18:3(cis-9,12,15) Alpha-Linolenic acid & $<\mathrm{LOQ}$ & $15.4 \pm 8.6$ & $11.5 \pm 1.1$ & $16.6 \pm 4.4^{*}$ \\
\hline $\begin{array}{l}\text { C20:4(cis-5,8,11,14) Eicosa- } \\
\text { 5,8,11,14,17-pentaenoic acid }\end{array}$ & $<\mathrm{LOQ}$ & 9,9 & $10.9 \pm 2.5$ & 13,6 \\
\hline $\begin{array}{l}\text { C22:6(cis-4,7,10,13,16,19) cis- } \\
4,7,10,13,16,19 \text {-Docosahexaenoic acid }\end{array}$ & $25.4 \pm 7.5$ & $38.2 \pm 8.2$ & $21.7 \pm 7.6$ & $30.4 \pm 6.8^{*}$ \\
\hline$n-6 / n-3$ PUFA & 8.30 & 11.20 & 10.70 & 7.90 \\
\hline
\end{tabular}

Values are presented as mean $\pm \mathrm{SD}$.

${ }^{*} P<0.05$ before vs. after within each group (control or $\mathrm{n}-3 \mathrm{PUFA}$ ); ${ }^{\dagger} P<0.05$ difference between the groups.

PUFA, polyunsaturated fatty acid; SFA, saturated fatty acid.

$<L O Q$, below the limit of quantification: C10:0 capric acid, C12:0 lauric acid, C13:0 tridecylic acid, C15:0 pentadecylic acid, C17:0 margaric acid, C20:0 arachidic acid, C22:0 behenic acid, C23:0 tricosanoic acid, C24:0 lignoceric acid, C14:1(cis-9) myristoleic acid, C20:1(cis-11) 11-eicosenoic acid, C22:1(cis-13) erucic acid, C24:1(cis-15) nervonic acid, C21:2(cis-11,14) eicosadienoic acid.

Not found: C4:0 butyric acid, C6:0 caproic acid, C8:0 caprylic acid, C11:0 undecylic acid, C21:0 heneicosanoic acid, C15:1(cis-10) cis-10-pentadecenoic acid, C17:1(cis-10) cis-10-heptadecenoic acid, C18:1(trans-9) elaidic acid, C18:2(trans-9,12) linoelaidic acid, C22:2(cis-13,16) 13,16-docosadienoic acid, C20:3(cis-11,14,17) 11,14,17-eicosatrienoic acid. 


\section{Serum fatty acid profiles}

The validity of the dietary protocol was confirmed by a significant increase in n-3 PUFA concentration in the serum of competitive athletes. The profiles of 37 fatty acids in serum before and after the dietary protocol is presented in Table 2. At baseline, there were no significant differences in serum fatty acids concentrations between the groups. At the end of dietary protocol vs. baseline, in the n-3 PUFAs group only serum concentrations of ALA and DHA were significantly increased, and the serum n- $6 / n-3$ ratio was decreased by approximately $27 \%$. For the control group, the serum fatty acids profile did not significantly change, although the $n-6 / n-3$ ratio increased by approximately $35 \%$. Serum C20:4(cis-5,8,11,14) arachidonic acid (AA) was significantly lower in the n-3 PUFA group compared with the control group after completion of each dietary protocol.

\section{Forearm skin microvascular reactivity - endothelium-dependent and -independent vasodilation} Consumption of n-3 PUFA-enriched hen eggs significantly enhanced both PORH (an increase of 17\%; Fig. 2A) and AChID (an increase of 14.4\%; Fig. 2B) of forearm skin microcirculation compared with baseline measurements. However, consumption of regular hen eggs did not induce any significant changes in PORH or AChID vs. baseline (Fig. 2A and 2B), and there was no significant difference in PORH and AChID at baseline or following consumption of eggs between the groups (Fig. 2A and 2B). SNPID was similar between baseline and following consumption of regular or n-3 PUFA eggs within each group (Fig. 2C), and between control and n-3 PUFA groups at baseline and following each diet protocol (Fig. 2C).

\section{Microvascular responsiveness range to AEE}

Consumption of n-3 PUFA-enriched hen eggs significantly increased the $\triangle \mathrm{AChID}$ responsiveness range to $\mathrm{AEE}$ (Fig. 3B), while there was no significant effect on $\triangle \mathrm{PORH}$ (Fig. 3A) or $\triangle$ SNPID (Fig. 3C) responsiveness to AEE. In addition, consumption of regular hen eggs did not induce any significant changes in $\triangle \mathrm{PORH}$ (Fig. $3 \mathrm{~A}$ ), $\triangle \mathrm{AChID}$ (Fig. 3B), or $\triangle$ SNPID (Fig. 3C) responsiveness to AEE session compared with baseline. There was no significant difference in $\triangle \mathrm{PORH}, \triangle \mathrm{AChID}$, or $\triangle \mathrm{SNP}$ at baseline or following eggs consumption between groups (Fig. 3).

\section{Markers of oxidative stress and antioxidant defense}

Plasma TBARS (marker of oxidative stress and lipid peroxidation) and FRAP (marker of antioxidant defense) levels and serum antioxidant enzyme (CAT, GPx, and SOD) activities in group before and after the dietary protocol are presented in Table 3. Both TBARS and FRAP were similar before and after dietary protocol within each group, and did not differ between control and n-3 PUFAs groups at baseline or following each respective diet protocol. Serum activity of GPx and SOD significantly decreased, whereas activity of CAT remained unchanged following consumption of n-3 PUFA-enriched hen eggs vs. baseline. Activity of all measured antioxidant enzymes (CAT, GPx, and SOD) were similar before and after the dietary protocol within the control group. Furthermore, serum CAT, GPx, and SOD activities did not significantly differ between the control and n-3 PUFAs groups at baseline or following each respective diet protocol.

\section{DISCUSSION}

This was the first randomized double-blinded placebo-
A

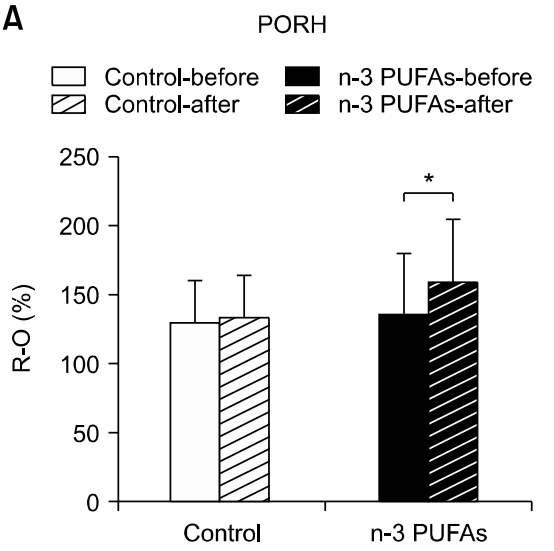

B
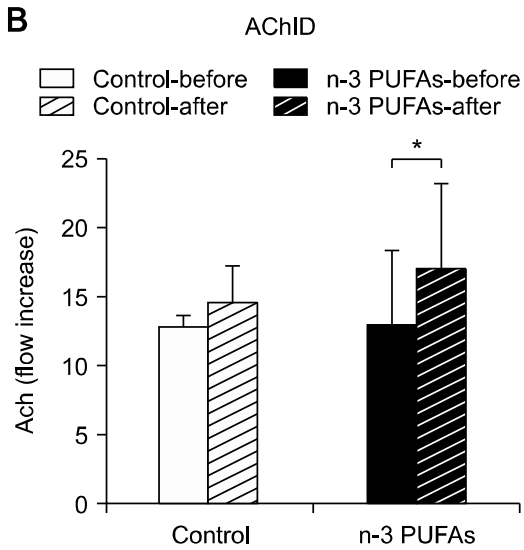

C
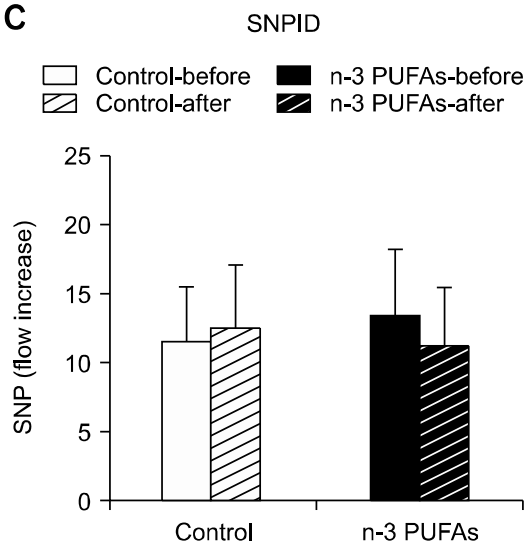

Fig. 2. Effect of regular (control group, $n=9$ ) and $n-3$ polyunsaturated fatty acid (PUFA)-enriched ( $n-3$ PUFAs group, $n=14$ ) hen egg consumption on skin microvascular overall reactivity, and endothelium-dependent and -independent vasodilation in competitive athletes. (A) Post-occlusive reactive hyperemia (PORH), (B) acetylcholine-induced dilation (AChID), and (C) sodium nitroprusside-induced dilation (SNPID). PORH measurements are expressed as the difference between percentages of flow change during reperfusion and occlusion in relation to baseline (R-0\%). AChID and SNPID are expressed as flow increases following ACh or SNP administration compared with baseline flow. Data are presented as arithmetic mean \pm SD. ${ }^{*} P=0.002$ (for $\mathrm{PORH}$ ) and $P=0.050$ (for AChID) before vs. after within the n-3 PUFA group (paired $t$-tests). 
A

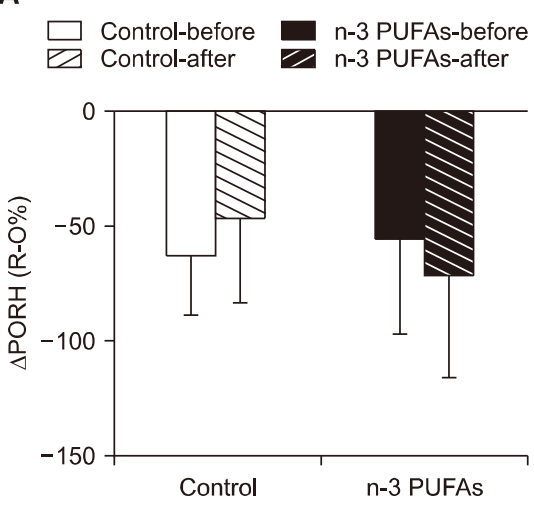

B

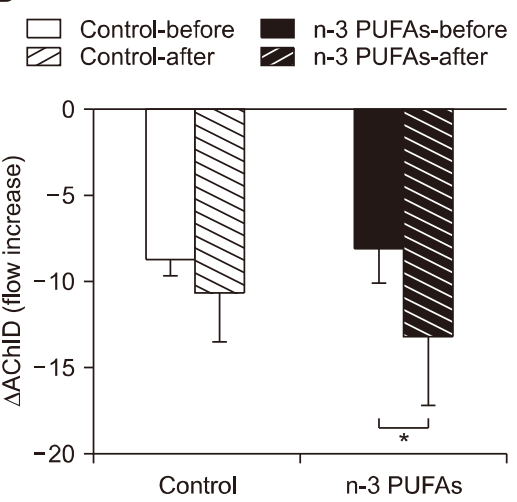

C
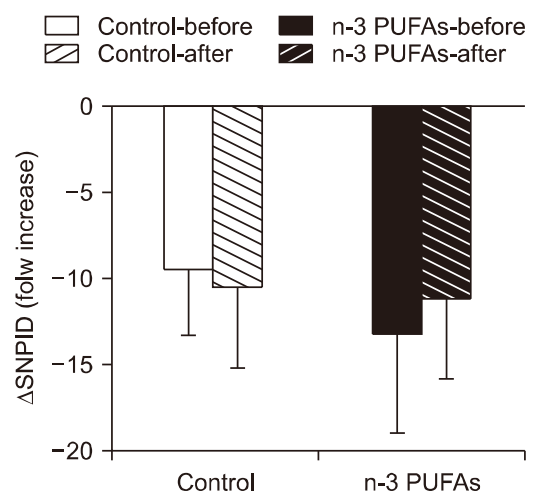

Fig. 3. Effect of regular (control group, $n=9$ ) and $n-3$ polyunsaturated fatty acid (PUFA)-enriched ( $n-3$ PUFA group, $n=14$ ) hen egg consumption on skin microvascular responsiveness range to acute exhausting exercise (AEE) sessions in competitive athletes. (A) Difference in post occlusive reactive hyperemia $(P O R H)$ responsiveness, $\triangle P O R H=P O R H_{\text {post-AEE }}-P O R H_{\text {pre-AEE, }}(B)$ difference in acetylcholine-induced dilation (AChID) responsiveness, $\triangle A C h I D=A C h I D_{\text {post- } A E E}-A_{C h I D} D_{\text {pre-AEE, }}$ and $(C)$ difference in sodium nitroprusside-induced dilation (SNPID) responsiveness, $\triangle$ SNPID=SNPID post-AEE $_{-}$SNPID $D_{\text {pre-AEE. }}$ PORH measurements are expressed as the difference between percentage of flow change during reperfusion and occlusion in relation to baseline (R-0\%). AChID and SNPID are expressed as flow increase following ACh or SNP administration compared to baseline flow. Data are presented as arithmetic mean \pm SD. ${ }^{*} P=0.008$ (for $\triangle$ AChID) before vs. after within the $\mathrm{n}-3$ PUFA group (paired $t$-test).

controlled interventional study to investigate if supplementation with n-3 PUFA functional foods had beneficial effects additive to exercise on endothelial function in competitive athletes. In this study, we showed that consumption of n-3 PUFA-enriched hen eggs significantly improved endothelium-dependent vasodilation of forearm skin microcirculation in response to vascular occlusion and administration of ACh, independently of BP and serum lipids changes (no correlation was found between primary outcomes with any of measured variables). Moreover, functional vascular changes were accompanied by significant decreases in activities of antioxidant enzymes (GPx and SOD) in serum, potentially due to improved oxidative stress balances, which diminishes the need for antioxidant defense. Interestingly, consumption of n-3 PUFA-enriched hen eggs also modified forearm skin microvascular adaptation to AEE challenge in competitive athletes. The physiological purpose and mechanisms that mediate this effect pose an intriguing question for future functional vascular research in athletes. Furthermore, consumption of large amounts of regular hen eggs ( 3 eggs per day for 3 weeks) did not have a detrimental effect on vascular function, BP, serum fatty acids, or lipid profiles in athletes, similar to previous observations (Stupin et al., 2018a; Stupin et al., 2020).

\section{n-3 PUFA-enriched hen egg consumption and microvascular endothelium-dependent vasodilation}

In recent years, n-3 PUFAs have received increasing attention in sports nutrition. n-3 PUFAs have been considered as ergogenic supplements, which may have a role in preparation for exercise, improving exercise efficiency, enhancing recovery from exercise, and helping to prevent injury during intense training. This may help reduce exercise-induced inflammation and improve muscle health of muscle and energy availability (Simopoulos, 2008; Ochi and Tsuchiya, 2018). In a previous study, compared with placebo, $1.3 \mathrm{~g}$ of n-3 PUFA significantly increased flowmediated dilation of brachial arteries in endurancetrained athletes, and induced increased levels of nitric

Table 3. The effect of regular (control group) and n-3 PUFA-enriched ( $n-3$ PUFAs group) hen egg consumption on oxidative stress levels, antioxidant defense, and antioxidant enzymes activity

\begin{tabular}{|c|c|c|c|c|}
\hline \multirow{2}{*}{ Parameter } & \multicolumn{2}{|c|}{ Control } & \multicolumn{2}{|c|}{ n-3 PUFAs } \\
\hline & Before & After & Before & After \\
\hline TBARS (mM/L TE) & $0.30 \pm 0.07$ & $0.31 \pm 0.05$ & $0.35 \pm 0.15$ & $0.35 \pm 0.10$ \\
\hline FRAP $(\mu \mathrm{m} / \mathrm{MDA})$ & $0.33 \pm 0.04$ & $0.40 \pm 0.11$ & $0.33 \pm 0.04$ & $0.33 \pm 0.04$ \\
\hline CAT (U/mg protein) & $3.511 \pm 2.417$ & $3.624 \pm 1.082$ & $2.439 \pm 1.381$ & $3.059 \pm 0.986$ \\
\hline GPx (U/mg protein) & $0.012 \pm 0.010$ & $0.009 \pm 0.004$ & $0.012 \pm 0.004$ & $0.006 \pm 0.003^{*}$ \\
\hline SOD (U/mg protein) & $8.964 \pm 0.624$ & $8.634 \pm 0.883$ & $9.289 \pm 0.581$ & $8.318 \pm 0.853^{*}$ \\
\hline
\end{tabular}

Data are presented as mean \pm SD.

${ }^{*} P<0.05$ before vs. after within each group (control or $n-3$ PUFA).

PUFA, polyunsaturated fatty acid; TBARS, thiobarbituric acid reactive substances; FRAP, ferric reducing ability of plasma; TE, Trolox equivalent; MDA, malondialdehyde; CAT, catalase; GPx, glutathione peroxidase; SOD, superoxide dismutase. 
oxide (NO) vs. pre-supplementation (Żebrowska et al., 2015). However, very few interventional studies have investigated the effects of n-3 PUFA supplementation on microvascular function (none in athletes), and those that exist show conflicting results (Armah et al., 2008; Fuentes et al., 2008). The present study demonstrated, for the first time, that peripheral microvascular response to vascular occlusion (PORH) and iontophoresis of $\mathrm{ACh}$ were enhanced in athletes who consumed n-3 PUFA-enriched hen eggs, which is consistent with previous results in sedentary persons (Kvernmo et al., 1998; Jurva et al., 2006; Stupin et al., 2018b).

It has been established that EPA and DHA compete with AA for incorporation into phospholipidic cell membranes, helping to decrease chronic and acute inflammation via inflammation mediators such as prostaglandins, leukotrienes, lipoxins, resolvins, and protectins (RamosCampo et al., 2020). Moreover, n-3 PUFAs can undergo enzymatic reactions that produce lipid mediators, and interact with oxygen free radicals (Yang et al., 2019). Therefore, n-3 PUFAs may have an important impact on vascular function since metabolites of AA [cyclooxygenase (COX) and cytochrome P450 (CYP450) derived vasoactive substances] and NO are key mediators of endothelium-dependent vasodilation and vasoconstriction (Drenjančević et al., 2016). Oxidative stress, inflammation and activation of the endothelium are key determinants of endothelial dysfunction. Therefore, the future research should establish which of these endothelium-derived vasoactive mediators (NO, COX, and/or CYP450derived) play a role in positive, endothelium-dependent vascular responses to n-3 PUFAs consumption in both healthy sedentary individuals and active athletes.

\section{n-3 PUFA-enriched hen eggs and microvascular responsiveness range to $A E E$}

Long-term regular exercise has beneficial effects on vascular function, resulting in improved macro- and microvascular endothelium-dependent vasodilation in physically active vs. sedentary individuals (Kvernmo et al., 1998; Lenasi and Strucl, 2004). However, the impact of the AEE on macro- and microvascular function is still under investigation. Our previous study demonstrated significantly reduced $\mathrm{PORH}$ and $\mathrm{ACh}$-induced skin microcirculation dilation following AEE in professional rowers who, prior to AEE sessions, exhibited enhanced baseline ACh-induced dilation vs. sedentary individuals (Stupin et al., 2018b). Similar results on the effects of AEE on microvascular function were observed in the present study, i.e., PORH and AChID were significantly reduced immediately after AEE vs. baseline. It is important to note that in the n-3 PUFA group, the temporary reduction in
AChID following AEE was more pronounced at the end of dietary protocol than at the beginning of the dietary protocol. This phenomenon was not observed in a group of athletes consuming regular eggs. From the point of the 'hormesis' hypothesis, if we consider that these temporary reductions in endothelial response led to better long-term endothelial function, a more pronounced reduction in AChID following n-3 PUFA supplementation could be considered as an increase in microvascular responsiveness range to AEE (i.e., better utilization of vasodilation capacity). This could potentially contribute to the enhanced baseline microvascular reactivity observed in athletes who consumed n-3 PUFA-enriched eggs. However, these results are just observational and we can only currently speculate of their physiological meaning.

\section{n-3 PUFA-enriched hen eggs, BP, and serum lipids}

In contrast to clinical trials on patients with increased CV risk or with $\mathrm{CV}$ diseases demonstrating clinically relevant reductions in BP with n-3 PUFA supplementation (Bønaa et al., 1990; Appel et al., 1993), unambiguous effects of n-3 PUFAs in lowering BP were not observed in normotensive individuals (Rasmussen et al., 2006; Shah et al., 2007). For example, reports have shown that consumption of $6 \mathrm{~g} / \mathrm{d}$ of DHA-rich fish oil for five weeks decreased resting diastolic BP in elite Australian Rules footballers (Buckley et al., 2009). However, consumption of $1.3 \mathrm{~g}$ of n-3 PUFA in capsules for 3 weeks did not induce significantly increase BP or HR compared with pre-supplementation levels in endurance-trained athletes (Żebrowska et al., 2015), which is similar to results of the present study. Since BP regulation may be influenced by many factors, it remains to be determined if n-3 PUFA consumption may modify these factors in healthy trained individuals.

The potential of n-3 PUFAs supplementation to reduce serum lipids, most notably triglycerides, in hyperlipidemic individuals is well accepted (Kris-Etherton et al., 2002; Jacobson et al., 2014). In addition, n-3 PUFA consumption has the ability to reduce serum triglyceride levels in healthy individuals (Leslie et al., 2015). Moreover, supplementation with n-3 PUFAs in the form of enriched hen eggs has potential to decrease serum triglycerides in healthy individuals (Oh et al., 1991; Bovet et al., 2007; Stupin et al., 2018a), judoists (Filaire et al., 2010), and in middle- and long-distance runners (Buonocore et al., 2020). However, in the present study, consumption of n-3 PUFA-enriched hen eggs did not induce significant changes in triglycerides levels in competitive athletes, potentially due to very favorable low (but normal) baseline serum triglycerides concentrations in athletes. 


\section{n-3 PUFAs and oxidative stress}

Intense bouts of acute exercise increase production of reactive oxygen species (ROS) and reactive nitrogen species (RNS), which leads to elevated oxidative stress and oxidative damage to cellular constituents (Powers and Jackson, 2008). ROS/RNS production induced by contracting muscles during exercise deplete cellular antioxidants (such as glutathione) in the blood, and alter the redox balance (Malhotra and Kaufman, 2007; Gammone et al., 2018). In general, regular exercise up-regulates the body's antioxidant defense mechanisms, to minimizing oxidative stress (Malhotra and Kaufman, 2007; Gammone et al., 2018). The effects of n-3 PUFAs in resolving oxidative stress in athletes are conflicting. For example, in 15 male athletes, drinking beverages enriched with DHA for 8 weeks increased the catalytic activity of SOD (but not CAT, glutathione reductase, or GPx), but did not alter other markers of oxidative stress (Martorell et al., 2015). Furthermore, supplementation with n-3 PUFAs for 8 weeks ( $4 \mathrm{~g} / \mathrm{d}$ ) significantly reduced the lipid peroxidation marker (MDA) and increased the activity of GPx and CAT, but not SOD, in middle- and long-distance running athletes (Buonocore et al., 2020). The present study demonstrated that consumption of n-3 PUFA-enriched hen eggs did not significantly change basal levels of lipid peroxidation and antioxidant capacity (FRAP) in competitive athletes. However, consumption decreased serum GPx and SOD activity, potentially due to the preexisting oxidative balance and adaptation of antioxidant mechanisms to exercise-induced increases in oxidative stress, while no changes in serum antioxidant enzyme activity were observed following consumption of regular hen eggs. These results may indicate athletes are better able to compensate for oxidative stress following n-3 PUFA supplementation. However, effect of n-3 PUFAs on oxidative stress levels immediately after exposure to the AEE sessions was not examined due to the technically demanding setup of the exercise; this effect needs to be clarified in future research.

This study has several limitations. First, there was a difference in sex distribution (a greater number of males than females) and only a limited number of included subjects, which underpowered the ability to examine potential differences between males and females. Furthermore, the participants' diets were not monitored throughout the study. However, to ensure maximum compliance to the dietary protocol, participants were frequently contacted by telephone and instructed to keep adhering to their usual diet.

In conclusion, this is the first time to show that consumption of n-3 PUFA-enriched functional foods improves peripheral endothelium-dependent microvascular reactivity, and decreases serum antioxidant enzyme (GPx and SOD) activity in competitive athletes. n-3 PUFA supplementation also beneficially modifies peripheral microvascular adaptations to the AEE challenge in competitive athletes. Enhanced microvascular function due to n-3 PUFA intake occurred independently of changes to BP and blood lipid concentrations, indicative of exciting new areas of research on the potential mechanisms mediating interaction between n-3 PUFAs, exercise, and the endothelium in athletes. The results of the current study could highlight recommendations for adequate n-3 PUFAs intake in athletes, particularly from natural sources such as functional foods.

\section{ACKNOWLEDGEMENTS}

This study is supported by the European Structural and Investment Funds grant for the Croatian National Scientific Center of Excellence for Personalized Health Care, University of Josip Juraj Strossmayer Osijek (grant no. KK.01.1.1.01.0010).

\section{AUTHOR DISCLOSURE STATEMENT}

The authors declare no conflict of interest.

\section{REFERENCES}

Appel LJ, Miller ER 3rd, Seidler AJ, Whelton PK. Does supplementation of diet with 'fish oil' reduce blood pressure? A metaanalysis of controlled clinical trials. Arch Intern Med. 1993. 153:1429-1438.

Armah CK, Jackson KG, Doman I, James L, Cheghani F, Minihane AM. Fish oil fatty acids improve postprandial vascular reactivity in healthy men. Clin Sci. 2008. 114:679-686.

Arterburn LM, Hall EB, Oken H. Distribution, interconversion, and dose response of n-3 fatty acids in humans. Am J Clin Nutr. 2006. 83:1467S-1476S.

Barić L, Drenjančević I, Matić A, Stupin M, Kolar L, Mihaljević Z, et al. Seven-day salt loading impairs microvascular endothelium-dependent vasodilation without changes in blood pressure, body composition and fluid status in healthy young humans. Kidney Blood Press Res. 2019. 44:835-847.

Barić L, Drenjančević I, Mihalj M, Matić A, Stupin M, Kolar L, et al. Enhanced antioxidative defense by vitamins $\mathrm{C}$ and $\mathrm{E}$ consumption prevents 7-day high-salt diet-induced microvascular endothelial function impairment in young healthy individuals. J Clin Med. 2020. 9:843. https://doi.org/10.3390/jcm9030843

Bønaa KH, Bjerve KS, Straume B, Gram IT, Thelle D. Effect of eicosapentaenoic and docosahexaenoic acids on blood pressure in hypertension. A population-based intervention trial from the Troms $\varnothing$ study. N Engl J Med. 1990. 322:795-801.

Borg GA. Psychophysical bases of perceived exertion. Med Sci Sports Exerc. 1982. 14:377-381.

Bovet P, Faeh D, Madeleine G, Viswanathan B, Paccaud F. Decrease in blood triglycerides associated with the consumption of eggs of hens fed with food supplemented with fish oil. Nutr Metab Cardiovasc Dis. 2007. 17:280-287.

Buckley JD, Burgess S, Murphy KJ, Howe PR. DHA-rich fish oil 
lowers heart rate during submaximal exercise in elite Australian Rules footballers. J Sci Med Sport. 2009. 12:503-507.

Buonocore D, Verri M, Giolitto A, Doria E, Ghitti M, Dossena M. Effect of 8-week n-3 fatty-acid supplementation on oxidative stress and inflammation in middle- and long-distance running athletes: a pilot study. J Int Soc Sports Nutr. 2020. 17:55. https://doi.org/10.1186/s12970-020-00391-4

Calder PC. n-3 polyunsaturated fatty acids, inflammation, and inflammatory diseases. Am J Clin Nutr. 2006. 83:1505S-1519S.

Cavka A, Cosic A, Jukic I, Jelakovic B, Lombard JH, Phillips SA, et al. The role of cyclo-oxygenase- 1 in high-salt diet-induced microvascular dysfunction in humans. J Physiol. 2015. 593: 5313-5324.

Chen MJ, Fan X, Moe ST. Criterion-related validity of the Borg ratings of perceived exertion scale in healthy individuals: a meta-analysis. J Sports Sci. 2002. 20:873-899.

Cosic A, Jukic I, Stupin A, Mihalj M, Mihaljevic Z, Novak S, et al. Attenuated flow-induced dilatation of middle cerebral arteries is related to increased vascular oxidative stress in rats on a short-term high salt diet. J Physiol. 2016. 594:4917-4931.

Dawson EA, Green DJ, Cable NT, Thijssen DH. Effects of acute exercise on flow-mediated dilatation in healthy humans. J Appl Physiol. 2013. 115:1589-1598.

Drenjančević I, Jukić I, Stupin A, Ćosić A, Stupin M, SelthoferRelatic K. The markers of endothelial activation. In: Lenasi $\mathrm{H}$, editor. Endothelial Dysfunction: Old Concepts and New Challenges. IntechOpen, London, UK. 2018 [cited 2021 Jun 15]. Available from: https://www.intechopen.com/chapters/60875

Drenjančević I, Kralik G, Kralik Z, Mihalj M, Stupin A, Novak S, et al. The effect of dietary intake of omega-3 polyunsaturated fatty acids on cardiovascular health: revealing potentials of functional food. In: Shiomi N, Waisundara V, editors. Superfood and Functional Food: The Development of Superfoods and Their Roles as Medicine. IntechOpen, London, UK. 2017. [cited 2021 Jun 15]. Available from: https://www.intechopen. com/chapters/53687

Egert S, Baxheinrich A, Lee-Barkey YH, Tschoepe D, Wahrburg U, Stratmann B. Effects of an energy-restricted diet rich in plantderived $\alpha$-linolenic acid on systemic inflammation and endothelial function in overweight-to-obese patients with metabolic syndrome traits. Br J Nutr. 2014. 112:1315-1322.

Filaire E, Massart A, Portier H, Rouveix M, Rosado F, Bage AS, et al. Effect of 6 weeks of n-3 fatty-acid supplementation on oxidative stress in Judo athletes. Int J Sport Nutr Exerc Metab. 2010. 20:496-506.

Fuentes F, López-Miranda J, Pérez-Martínez P, Jiménez Y, Marín C, Gómez P, et al. Chronic effects of a high-fat diet enriched with virgin olive oil and a low-fat diet enriched with alphalinolenic acid on postprandial endothelial function in healthy men. Br J Nutr. 2008. 100:159-165.

Gammone MA, Riccioni G, Parrinello G, D’Orazio N. Omega-3 polyunsaturated fatty acids: benefits and endpoints in sport. Nutrients. 2018. 11:46. https://doi.org/10.3390/nu11010046

Jacobson TA, Ito MK, Maki KC, Orringer CE, Bays HE, Jones PH, et al. National Lipid Association recommendations for patientcentered management of dyslipidemia: part 1-executive summary. J Clin Lipidol. 2014. 8:473-488.

Joyner MJ, Green DJ. Exercise protects the cardiovascular system: effects beyond traditional risk factors. J Physiol. 2009. 587: 5551-5558.

Jurva JW, Phillips SA, Syed AQ, Syed AY, Pitt S, Weaver A, et al. The effect of exertional hypertension evoked by weight lifting on vascular endothelial function. J Am Coll Cardiol. 2006. 48: 588-589.

Kris-Etherton PM, Harris WS, Appel LJ; American Heart Association. Nutrition Committee. Fish consumption, fish oil, omega-3 fatty acids, and cardiovascular disease. Circulation.
2002. 106:2747-2757.

Kvernmo HD, Stefanovska A, Kirkebøen KA, Osterud B, Kvernebo K. Enhanced endothelium-dependent vasodilatation in human skin vasculature induced by physical conditioning. Eur J Appl Physiol Occup Physiol. 1998. 79:30-36.

Lenasi H, Strucl M. Effect of regular physical training on cutaneous microvascular reactivity. Med Sci Sports Exerc. 2004. 36: 606-612.

Leslie MA, Cohen DJ, Liddle DM, Robinson LE, Ma DW. A review of the effect of omega-3 polyunsaturated fatty acids on blood triacylglycerol levels in normolipidemic and borderline hyperlipidemic individuals. Lipids Health Dis. 2015. 14:53. https:// doi.org/10.1186/s12944-015-0049-7

Malhotra JD, Kaufman RJ. Endoplasmic reticulum stress and oxidative stress: a vicious cycle or a double-edged sword? Antioxid Redox Signal. 2007. 9:2277-2293.

Martorell M, Capó X, Bibiloni MM, Sureda A, Mestre-Alfaro A, Batle JM, et al. Docosahexaenoic acid supplementation promotes erythrocyte antioxidant defense and reduces protein nitrosative damage in male athletes. Lipids. 2015. 50:131-148.

Mihalj M, Stupin A, Kolobarić N, Bujak IT, Matić A, Kralik Z, et al. Leukocyte activation and antioxidative defense are interrelated and moderately modified by $n-3$ polyunsaturated fatty acid-enriched eggs consumption-double-blind controlled randomized clinical study. Nutrients. 2020. 12:3122. https://doi. org/10.3390/nu12103122

Mihaljević Z, Matić A, Stupin A, Rašić L, Jukić I, Drenjančević I. Acute hyperbaric oxygenation, contrary to intermittent hyperbaric oxygenation, adversely affects vasorelaxation in healthy Sprague-Dawley rats due to increased oxidative stress. Oxid Med Cell Longev. 2018. 2018:7406027. https://doi.org/10. $1155 / 2018 / 7406027$

Morgan DR, Dixon LJ, Hanratty CG, El-Sherbeeny N, Hamilton PB, McGrath LT, et al. Effects of dietary omega-3 fatty acid supplementation on endothelium-dependent vasodilation in patients with chronic heart failure. Am J Cardiol. 2006. 97:547551.

Ochi E, Tsuchiya Y. Eicosapentaenoic acid (EPA) and docosahexaenoic acid (DHA) in muscle damage and function. Nutrients. 2018. 10:552. https://doi.org/10.3390/nu10050552

Oh SY, Ryue J, Hsieh CH, Bell DE. Eggs enriched in omega-3 fatty acids and alterations in lipid concentrations in plasma and lipoproteins and in blood pressure. Am J Clin Nutr. 1991. 54: 689-695.

Oliver JM. Omega-3 fatty acids and student-athletes: is it time for better education and a policy change?. J Athl Train. 2019. 54: 5-6.

Powers SK, Jackson MJ. Exercise-induced oxidative stress: cellular mechanisms and impact on muscle force production. Physiol Rev. 2008. 88:1243-1276.

Ramos-Campo DJ, Ávila-Gandía V, López-Román FJ, Miñarro J, Contreras C, Soto-Méndez F, et al. Supplementation of re-esterified docosahexaenoic and eicosapentaenoic acids reduce inflammatory and muscle damage markers after exercise in endurance athletes: a randomized, controlled crossover trial. Nutrients. 2020. 12:719. https://doi.org/10.3390/nu12030719

Rasmussen BM, Vessby B, Uusitupa M, Berglund L, Pedersen E, Riccardi G, et al.; KANWU Study Group. Effects of dietary saturated, monounsaturated, and n-3 fatty acids on blood pressure in healthy subjects. Am J Clin Nutr. 2006. 83:221-226.

Renier G, Skamene E, DeSanctis J, Radzioch D. Dietary n-3 polyunsaturated fatty acids prevent the development of atherosclerotic lesions in mice. Modulation of macrophage secretory activities. Arterioscler Thromb. 1993. 13:1515-1524.

Ritz PP, Rogers MB, Zabinsky JS, Hedrick VE, Rockwell JA, Rimer EG, et al. Dietary and biological assessment of the omega-3 status of collegiate athletes: a cross-sectional analysis. PLoS 
One. 2020. 15:e0228834. https://doi.org/10.1371/journal. pone. 0228834

Shah AP, Ichiuji AM, Han JK, Traina M, El-Bialy A, Meymandi SK, et al. Cardiovascular and endothelial effects of fish oil supplementation in healthy volunteers. J Cardiovasc Pharmacol Ther. 2007. 12:213-219.

Simopoulos AP. Omega-3 fatty acids, exercise, physical activity and athletics. In: Simopoulos AP, editor. Nutrition and Fitness: Cultural, Genetic and Metabolic Aspects. Karger, Basel, Switzerland. 2008. p 23-50.

Stupin A, Mihalj M, Kolobarić N, Šušnjara P, Kolar L, Mihaljević $Z$, et al. Anti-inflammatory potential of $n-3$ polyunsaturated fatty acids enriched hen eggs consumption in improving microvascular endothelial function of healthy individuals - clinical trial. Int J Mol Sci 2020. 21:4149. https://doi.org/10.3390/ ijms21114149

Stupin A, Rasic L, Matic A, Stupin M, Kralik Z, Kralik G, et al. Omega-3 polyunsaturated fatty acids-enriched hen eggs consumption enhances microvascular reactivity in young healthy individuals. Appl Physiol Nutr Metab. 2018a. 43:988-995.

Stupin M, Kibel A, Stupin A, Selthofer-Relatić K, Matić A, Mihalj $M$, et al. The physiological effect of n-3 polyunsaturated fatty acids (n-3 PUFAs) intake and exercise on hemorheology, microvascular function, and physical performance in health and cardiovascular diseases; is there an interaction of exercise and dietary n-3 PUFA intake?. Front Physiol. 2019. 10:1129. https://doi.org/10.3389/fphys.2019.01129

Stupin M, Stupin A, Rasic L, Cosic A, Kolar L, Seric V, et al. Acute exhaustive rowing exercise reduces skin microvascular dilator function in young adult rowing athletes. Eur J Appl Physiol. 2018b. 118:461-474.
Thielecke F, Blannin A. Omega-3 fatty acids for sport performance - are they equally beneficial for athletes and amateurs? A narrative review. Nutrients. 2020. 12:3712. https://doi.org/ 10.3390/nu12123712

Thies F, Garry JM, Yaqoob P, Rerkasem K, Williams J, Shearman $\mathrm{CP}$, et al. Association of n-3 polyunsaturated fatty acids with stability of atherosclerotic plaques: a randomised controlled trial. Lancet. 2003. 361:477-485.

Thorlaksdottir AY, Skuladottir GV, Petursdottir AL, Tryggvadottir L, Ogmundsdottir HM, Eyfjord JE, et al. Positive association between plasma antioxidant capacity and n-3 PUFA in red blood cells from women. Lipids. 2006. 41:119-125.

von Schacky C, Kemper M, Haslbauer R, Halle M. Low omega-3 index in 106 German elite winter endurance athletes: a pilot study. Int J Sport Nutr Exerc Metab. 2014. 24:559-564.

Wilson PB, Madrigal LA. Associations between whole blood and dietary omega-3 polyunsaturated fatty acid levels in collegiate athletes. Int J Sport Nutr Exerc Metab. 2016. 26:497-505.

Yang B, Fritsche KL, Beversdorf DQ, Gu Z, Lee JC, Folk WR, et al. Yin-yang mechanisms regulating lipid peroxidation of docosahexaenoic acid and arachidonic acid in the central nervous system. Front Neurol. 2019. 10:642. https://doi.org/10.3389/ fneur.2019.00642

Żebrowska A, Mizia-Stec K, Mizia M, Gąsior Z, Poprzęcki S. Omega-3 fatty acids supplementation improves endothelial function and maximal oxygen uptake in endurance-trained athletes. Eur J Sport Sci. 2015. 15:305-314.

Zehr KR, Walker MK. Omega-3 polyunsaturated fatty acids improve endothelial function in humans at risk for atherosclerosis: a review. Prostaglandins Other Lipid Mediat. 2018. 134:131140. 